Silvia Hansen-Schirra (Germersheim)/ Silke Gutermuth (Germersheim)

\title{
Multimodale Daten für die empirische Analyse grammatischer Variation in Übersetzungen
}

\begin{abstract}
Mögliche Erklärungshorizonte für grammatische Variation in Übersetzungen können durch kontrastive Unterschiede sowie Textsortenkonventionen für die involvierten Sprachen hergeleitet werden. Weiterhin ausschlaggebend sind die vom Übersetzer verwendeten Übersetzungsstrategien, wie Simplifizierung und Explizierung, die mit Methoden der Korpuslinguistik und der Translationsprozessforschung untersucht werden können. Letztere betreffend liefert das Eyetracking Hinweise auf Problemstellen im Ausgangstext; das Keylogging lässt Rückschlüsse auf die Problemlösestrategien im Zieltext zu. Durch die Triangulation der gewonnenen Produkt- und Prozessdaten kann einerseits der ganzheitliche Übersetzungsprozess und andererseits die Produktion der grammatischen Variation empirisch aufgearbeitet werden.
\end{abstract}

\section{Einleitung}

Durch die Verwendung bilingualer Übersetzungskorpora sowie monolingualer Vergleichskorpora von übersetzten und nicht-übersetzten Texten in der Zielsprache konnten erstmalig Theorien und Hypothesen in Bezug auf Übersetzungsstrategien und -universalien auf empirischer Basis überprüft werden. So haben Olohan/Baker (2000) fiktionale Texte im Translational English Corpus und im British National Corpus verglichen und nachgewiesen, dass englische Übersetzungen im Hinblick auf optionale Elemente expliziter sind als Originale im Englischen. Diese Art der Explizierung geht oftmals einher mit einer strukturellen Simplifizierung von Übersetzungen, indem beispielsweise finite Strukturen infiniten vorgezogen werden, und verursacht dadurch grammatische Veränderungen im Zieltext. Außerdem hat Hansen-Schirra (2008) auf der Basis eines englisch-deutschen Übersetzungskorpus gezeigt, dass die Äquivalenzforderungen auf der semantischen Ebene Vorrang vor denen auf der strukturellen Ebene haben, was wiederum zu grammatischen Variationen in Zieltexten führen kann. Diese Übersetzungsshifts sind einerseits durch kontrastive Unterschiede zwischen den involvierten Sprachen bedingt, andererseits aber auch durch unterschiedliche Textsortenkonventionen bzw. Registerwechsel. 
Nun lassen die korpuslinguistischen Ergebnisse zwar Rückschlüsse auf unterschiedliche Frequenzeffekte zwischen Ausgangs- und Zieltexten zu, oftmals bleiben die Zusammenhänge und Ursachen für die grammatischen Verschiebungen aber unklar, vor allem wenn sie auf Übersetzungsstrategien und kognitive Prozesse beim Übersetzen zurückzuführen sind. Um dadurch bedingte grammatische Veränderungen empirisch untersuchen zu können, ist eine multi-methodische und multimodale Herangehensweise notwendig. Daher werden Produktund Prozessdaten miteinander trianguliert, um ein holistisches Bild des Übersetzungsprozesses zu erhalten (Carl/Jakobsen/Hvelplund Jensen 2008). Im Folgenden sollen exemplarisch Methoden diskutiert werden, mit denen man diese Prozesse näher beleuchten und die Variation in Übersetzungen nicht nur messen, sondern auch Erklärungsvorschläge dafür liefern kann.

\section{Grammatische Variation in Übersetzungskorpora}

Grammatische Variation kann in Übersetzungen auf zwei Arten in Erscheinung treten. Einerseits kann die Übersetzung im Vergleich zum Ausgangstext variant sein. Parallele Übersetzungskorpora, bestehend aus ausgangssprachlichen Texten und deren Übersetzungen in der Zielsprache, werden hierfür einer Analyse unterzogen. Andererseits kann die Variation aber auch zwischen Übersetzungen und originalsprachlichen Texten in der Zielsprache auftreten. Um dies zu quantifizieren, werden monolinguale Vergleichskorpora, bestehend aus übersetztem und nicht-übersetztem Text in der Zielsprache, kontrastiert (siehe Baker 1996 für eine detailliertere Definition).

Die empirische Analyse grammatischer Variation in Übersetzungen setzt eine reichhaltige Korpusannotation sowie Alignierung der Übersetzungskorpora voraus. Das deutsch-englische CroCo-Korpus (Hansen-Schirra/Neumann/Steiner 2012) beinhaltet eine Treebank-Annotation (d.h. eine syntaktische Annotation, Brants et al. 2004) für alle Subkorpora (englische Originale, deutsche Originale, deutschenglische Übersetzungen und englisch-deutsche Übersetzungen) sowie Alignierung auf Satz-, Teilsatz-, Phrasen- und Wortebene.

Dies ermöglicht die Abfrage von grammatischen Strukturen und deren Vergleich in den Subkorpora. Sucht man beispielsweise nach allen Wörtern, die eine Alignierung aufweisen (die Alignierung erfolgt auf der Basis des semantischen Stammwortes), die allerdings unterschiedlichen Wortarten zugeordnet sind, erhält man die in den Übersetzungskorpora enthaltenen Wortartwechsel. Tabelle 1 
zeigt die Ergebnisse aus einer entsprechenden Abfrage für alle Inhaltswörter für die Textsorte Aktionärsbriefe im CroCo-Korpus (siehe auch Čulo et al. 2008).

Tab. 1: Variation durch Wortartenwechsel

\begin{tabular}{lcc}
\hline & Englisch-Deutsch & Deutsch-Englisch \\
\hline Verb-Nomen & $24,31 \%$ & $16,98 \%$ \\
Verb-Adjektiv & $11,69 \%$ & $2,80 \%$ \\
Verb-Adverb & $6,95 \%$ & $0,25 \%$ \\
Adjektiv-Nomen & $17,43 \%$ & $9,48 \%$ \\
Adjektiv-Verb & $1,84 \%$ & $9,92 \%$ \\
Adjektiv-Adverb & $1,42 \%$ & $11,58 \%$ \\
Nomen-Adjektiv & $13,89 \%$ & $21,63 \%$ \\
Nomen-Verb & $5,74 \%$ & $16,98 \%$ \\
Nomen-Adverb & $3,40 \%$ & $1,08 \%$ \\
Adverb-Adjektiv & $10,06 \%$ & $1,34 \%$ \\
Adverb-Nomen & $3,05 \%$ & $1,59 \%$ \\
Adverb-Verb & $0,21 \%$ & $6,36 \%$ \\
\hline
\end{tabular}

Tabelle 1 zeigt, dass die gewählten Übersetzungsstrategien für die Übersetzungsrichtung Englisch-Deutsch nominalisierende Variation bewirken (z.B. mehr VerbNomen, Verb-Adjektiv, Adverb-Adjektiv), wohingegen die Variation für deutschenglische Übersetzungen verbalisierend wirkt (z.B. Nomen-Verb). Die hier diskutierten Wortklassenwechsel werden in der Übersetzungswissenschaft als Transpositionen bezeichnet (vgl. Vinay/Darbelnet 1995; Newmark 1988).

Tab. 2: Variation im Vorfeld

\begin{tabular}{llllr}
\hline & Subjekt & Adverbiale & Objekt & Sonstige \\
\hline Originale EN & $63,43 \%$ & $27,14 \%$ & $0,15 \%$ & $9,28 \%$ \\
Übersetzung DE-EN & $64,20 \%$ & $27,13 \%$ & $0,19 \%$ & $8,48 \%$ \\
Übersetzung EN-DE & $55,47 \%$ & $36,08 \%$ & $2,42 \%$ & $6,03 \%$ \\
Originale DE & $50,25 \%$ & $31,00 \%$ & $8,46 \%$ & $10,29 \%$ \\
\hline
\end{tabular}

Tabelle 2 zeigt die Frequenzen der unterschiedlichen syntaktischen Funktionen, die im Vorfeld der Aussagesätze des Korpus auftreten. Vor dem Hintergrund der systemischen Unterschiede im Bereich der Wortstellung von englischen und deutschen Sätzen zeigt sich hier vor allem ein Unterschied bei den Objekten im Vorfeld. Das Englische zeichnet sich durch eine kanonische Wortstellung aus, wohin- 
gegen das Deutsche sehr viel mehr Freiheit zulässt (vgl. Hawkins 1986; König/Gast 2007). Daher sind Objekte im Vorfeld in der englischen Schriftsprache wegen der fixen SVO-Wortstellung in Aussagesätzen nicht möglich und auch bei den englisch-deutschen Übersetzungen unterrepräsentiert. Dies deutet auf eine eher wörtliche Übernahme der Wortstellung hin, was wiederum zu einer grammatischen Variation im Vergleich zu den deutschen Originalen führt.

In Bezug auf die Frequenzen der Subjekte und Objekte nähern sich jedoch die deutschen Übersetzungen den Zahlen der deutschen Originale an. Eine Erklärung hierfür könnte darin liegen, dass die für das Englische typische sekundäre Subjektivierung (vgl. Rohdenburg 1974; Krein-Kühle 2001) im Deutschen durch Adverbialien oftmals gefolgt von Passivierung realisiert wird, wie das folgende Beispiel aus dem CroCo-Korpus zeigt:

Original EN: $\quad$... textbooks often write this process as ...

Übersetzung DE: In Lehrbüchern wird dieser Prozeß oft durch ... dargestellt.

In diesem Beispiel wird das unbelebte Agens „textbooks“ in der Übersetzung durch ein Lokaladverbiale „In Lehrbüchern“ mit nachfolgender Passivierung realisiert. Die Übersetzung folgt den typischen Strukturen der Zielsprache, was Baker (1996) als Normalisierung und Toury (1995) als Standardisierung bezeichnet. Diese Art des Funktions- und Perspektivenwechsels wird in der Übersetzungswissenschaft unter dem Phänomen der Modulation subsumiert (vgl. Vinay/ Darbelnet 1995; Newmark 1988).

\section{Produktionsprozesse}

Um jedoch den Ausgangspunkt solcher Variationsphänomene in der Übersetzung verstehen zu können, reicht die Korpusanalyse nicht aus. Die Analyse des Übersetzungsprozesses und insbesondere der Produktionsprozesse im Zieltext können auf der Basis von Keylogging nachvollzogen werden. So wird beispielsweise durch das Protokoll der Tastatureingaben klar, wie Übersetzer mit kontrastiven Unterschieden und anderen Problemstellen umgehen bzw. ob grammatische Veränderungen im Sinne einer Normalisierung oder Explizierung vorgenommen werden. Exemplarisch soll dies am nachfolgenden Keylogging-Protokoll diskutiert werden (siehe auch Alves et al. 2010), das mit der Software Translog II aufgezeichnet wurde (Carl 2012).

Das Keylogging-Protokoll in Abbildung 1 zeigt den deutsch-englischen Übersetzungsentwurf des Satzes „Wir sind davon überzeugt, dass erfolgreiche Unternehmensführung und soziale Verantwortung sich nicht widersprechen." aus 
einem Marketingtext. Neben den Zeichen, die getippt werden, sieht man auch die Pausen (Sterne - bei längeren Pausen mit Sekundenangabe), die Löschungen (Rücktaste) und die Cursorbewegungen (Pfeile). Abbildung 2 zeigt die Revisionsphase für den gleichen Satz.

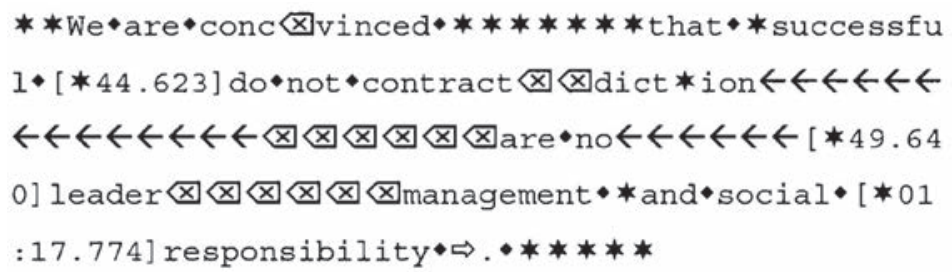

Abb. 1: Keylogging-Protokoll der Entwurfsphase

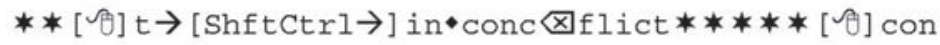 tradictory [ $* 26.575]$}

Abb. 2: Keylogging-Protokoll der Revisionsphase

In diesem Satz gibt es im Übersetzungs- und Revisionsprozess verschiedene grammatische Varianten für die Verbalphrase „sich nicht widersprechen“, die in Tabelle 3 schematisch dargestellt sind.

Tab. 3: Grammatische Varianten im Übersetzungs- und Revisionsprozess

\begin{tabular}{ll}
\hline Phase & Textversion \\
\hline Original DE & sich nicht widersprechen \\
Entwurf 1 & do not contradict \\
Entwurf 2 & do not contradiction \\
Entwurf 3 & are no contradiction \\
Revision 1 & are not in conflict \\
Revision 2 & are not contradictory \\
\hline
\end{tabular}

Man kann durch das Keylogging-Protokoll gut nachverfolgen, dass der Übersetzer in der Entwurfsphase zunächst die verbale Konstruktion als Rohübersetzung übernommen, sich dann aber für eine Nominalisierung entschieden hat. In der Revisionsphase kommt der Übersetzer nochmal auf diese Konstruktion zurück, setzt zunächst ein Synonym ein und entscheidet sich dann schlussendlich für eine Adjektivkonstruktion. 
Durch nähere Betrachtung der Keylogging-Daten fällt auf, dass die längeren Pausen dazu genutzt wurden, nach Synonymen in einem Online-Wörterbuch zu recherchieren. Weiterhin erklärt der Übersetzer beim retrospektiven Interview, dass er für den Marketingtext bewusst kein negatives Vokabular verwenden wollte und aus diesem Grund verschiedene Varianten ausprobiert und recherchiert hat und ihm am Ende die Adjektivkonstruktion am wenigsten negativ konnotiert erschien. Dieses Beispiel verdeutlicht einerseits das Potenzial der Methoden, wenn es um die Ermittlung der Übersetzungsvarianten (durch das Keylogging) sowie deren Charakterisierung und Gewichtung (durch das retrospektive Interview) geht. Andererseits wird gezeigt, dass die Berücksichtigung der Textsorte und des Registers bei der Übersetzung zu bewussten Übersetzungsstrategien und somit auch zu grammatischen Variationen im Zieltext führen kann.

\section{Verstehensprozesse}

Nachdem nun exemplarisch gezeigt wurde, wie die Produktionsprozesse näher beleuchtet werden können, soll abschließend der Frage nachgegangen werden, ob auch die Verstehensprozesse beim Übersetzen Variationsphänomene bewirken. Um dies zu untersuchen, wurde ein Experiment aufgesetzt (siehe Rabe 2012), in dem verschiedene Leseaufgaben miteinander verglichen wurden. Ein deutscher pharmazeutisch-medizinischer Fachtext (eine Packungsbeilage für ein Schnupfenspray) wurde von drei unterschiedlichen Probandengruppen gelesen: 26 Übersetzungsstudenten des MA-Studiengangs Translation sollten den Text zum reinen Textverständnis lesen. Eine Gruppe aus 21 Übersetzungsstudenten mit Medizin als Nebenfach sowie fünf professionelle medizinische Fachübersetzer erhielten den Auftrag, den Text als Vorbereitung auf die nachfolgende Übersetzung desselbigen zu lesen. Auf diese Weise konnten verschiedene Leseaufgaben (Lesen zum Textverstehen vs. Lesen als Übersetzungsvorbereitung) verglichen werden. Bei allen Probanden handelte es sich um deutsche Muttersprachler. Die Blickbewegungen während der Leseaufgaben wurden mit dem Eyetracker Tobii TX300 aufgezeichnet und mit der Software Tobii Studio ausgewertet.

Die Auswertung basiert auf der Grundannahme der so genannten „Eye-MindHypothese“ (Just/Carpenter 1980), dass Fixation und Informationsverarbeitung im Gehirn eng verknüpft sind. Studien aus der Leseforschung (Rayner 1998) zeigen, dass das Blickverhalten als Indikator für kognitiven Aufwand gesehen werden kann. Längere Fixationen können so auf Herausforderungen bei der Informationsverarbeitung hinweisen. 
Abbildung 3 zeigt die durchschnittliche Fixationsdauer in Sekunden pro Rezipientengruppe und Abbildung 4 die Anzahl der Fixationen für die drei unterschiedlichen Leseaufgaben. Obwohl es sich bei den Fachübersetzern um eine extrem kleine Probandengruppe handelt und aus diesem Grund keine statistischen Tests durchgeführt wurden, lassen beide Messwerte ähnliche Rückschlüsse zu: Lesen zum reinen Textverstehen erfordert die kürzeste Zeit und die wenigsten Fixationen. Beide Übersetzergruppen weisen längere Lesezeiten und mehr Fixationen beim Lesen als Vorbereitung zur Übersetzung auf als die Vergleichsgruppe beim Lesen ohne Übersetzungskontext, womit eine ähnliche Studie im Sprachenpaar Englisch-Dänisch von Jakobsen/Hvelplund Jensen (2008) bestätigt wird. Man kann also davon ausgehen, dass das Lesen in Vorbereitung auf eine Übersetzung Verstehensprozesse evoziert, die beim reinen Textverstehen keine Rolle spielen. Dies kann sich auf die Terminologie beziehen, auf die Fachsprachensyntax, auf semantische Relationen usw. Eine genauere Auswertung der Fixationen im Hinblick auf linguistische Ebenen (Terminologie, syntaktische Fachsprachenmerkmale etc.) und der Regressionen, d.h. der Textstellen, zu denen das Auge während des Lesens zurückspringt, soll in unserer künftigen Forschung weitere Schlussfolgerungen darüber zulassen, welche linguistischen Phänomene diese Prozessvariation verursacht.

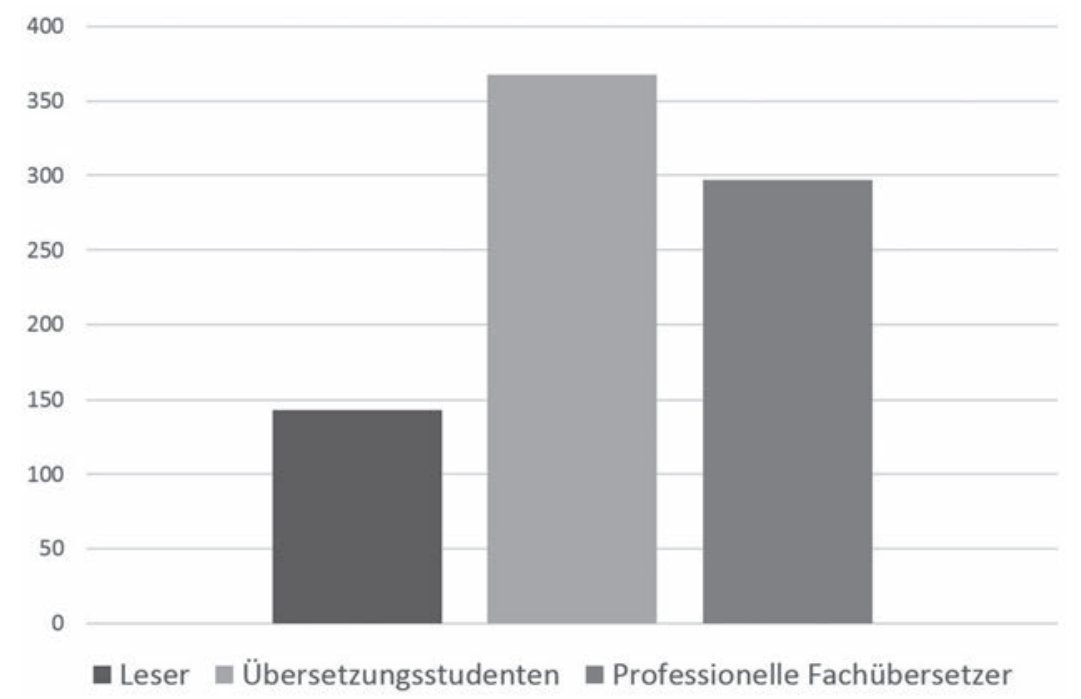

Abb. 3: Fixationsdauer (in Sekunden) 


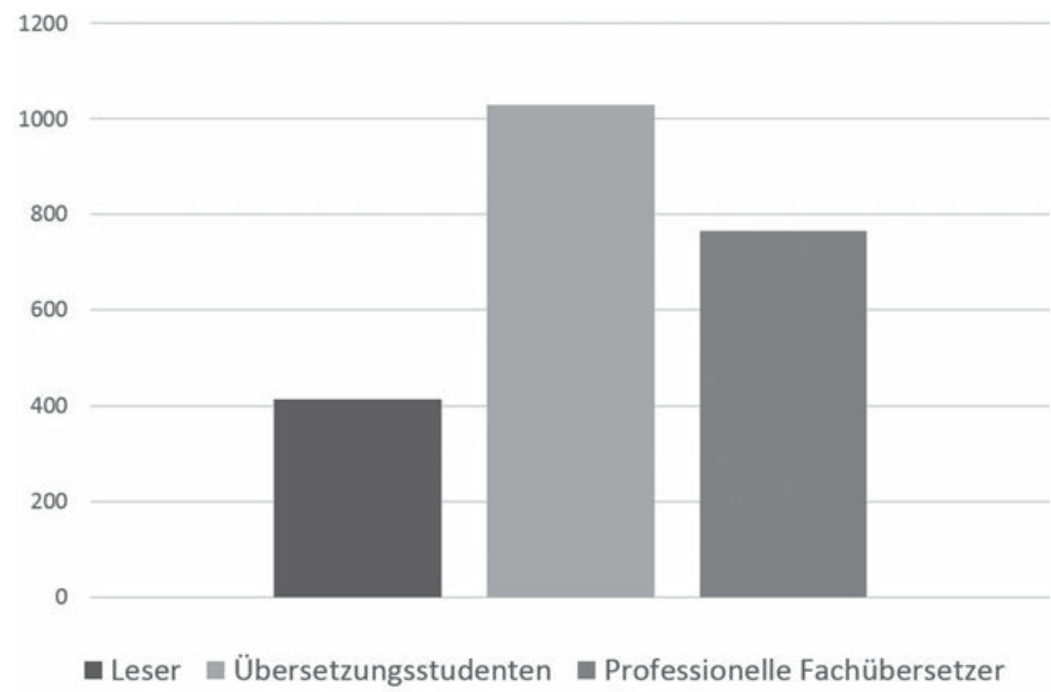

Abb. 4: Anzahl der Fixationen

Interessant ist jedoch zu beobachten, dass sich Verstehens- und Lesekompetenz mit zunehmender Erfahrung dahingehend entwickelt, dass sich bei den professionellen Fachübersetzern wieder die Lesedauer leicht verkürzt und die Anzahl der Fixationen leicht abnimmt. Dieses Phänomen innerhalb der Gruppe der Übersetzer ist ein Indiz dafür, dass sich translatorische Kompetenz - zumindest was den Lese- und Verstehensprozess angeht - quantifizieren lässt und messbar ist.

\section{Fazit und Ausblick}

Die bislang eher korpusanalytisch untersuchten Frequenzeffekte, wie Modulation und Transposition, können nur partiell zur Erklärung des Phänomens der grammatischen Variation in Übersetzungen beitragen. Um eine ganzheitliche Sichtweise der Entstehung dieser Variation zu erreichen, ist die hier vorgeschlagene multi-methodische und multimodale Herangehensweise notwendig. Sie schlägt den Bogen zu den kognitiven Prozessen beim Übersetzen, indem sie Lese- und Verstehensprozesse durch Eyetracking sowie Produktionsprozesse durch Keylogging transparent und quantifizierbar macht.

Es konnte gezeigt werden, dass die hier exemplarisch vorgestellten empirisch-multimodalen Daten im Hinblick auf die Entstehung, Messbarkeit und 
Erklärung der grammatischen Variation in Übersetzungen wichtige Erkenntnisse liefern können und dass sowohl Produktions- als auch Verstehensprozesse Variationsphänomene bewirken.

Zur Abrundung der Methodik wäre als möglicher nächster Schritt die Ausweitung der qualitativen Komponente, die hier durch retrospektive Interviews umgesetzt wurde, denkbar. Weitergehende Auswertungen der Eyetracking-Daten in Bezug auf Regressionen erlauben tiefer greifende Analysen der Verstehensprozesse; größere Probandengruppen könnten die Validität der Studie im Hinblick auf deren statistische Signifikanz, die bisher noch gar nicht getestet wurde, erhöhen. Damit könnte auch die Generalisierbarkeit der Phänomene im Sinne von universellen Übersetzungsprozessen oder die Beschränkung auf einzelne Sprachenpaarspezifika geklärt werden. Zudem bietet sich die Vertiefung des interessanten, aber noch wenig erforschten ,Nebenproduktes“ der Studie an: die Möglichkeit der empirischen Messung translatorischer Teilkompetenzen.

\section{Literatur}

Alves, Fabio et al. (2010): Translation units and grammatical shifts: Towards an integration of product- and process-based translation research. In: Shreve, Gregory M./Angelone, Erik (Hg.): Translation and cognition. (= American Translators Association Scholarly Monograph Series 15). Amsterdam, S. 109-142.

Baker, Mona (1996): Corpus-based translation studies: The challenges that lie ahead. In: Somers, Harold L. (Hg.): Terminology, LSP and translation: Studies in language engineering in honour of Juan C. Sager. (= Benjamins Translation Library 18). Amsterdam, S. 175-186.

Brants, Sabine et al. (2004): TiGer: Linguistic interpretation of a German corpus. In: Journal on Research on Language and Computation 2, S. 597-619.

Carl, Michael (2012): Translog-II: A program for recording user activity data for empirical reading and writing research. In: Proceedings of the Eighth International Conference on Language Resources and Evaluation, European Language Resources Association (ELRA). Istanbul, S. 4108-4112.

Carl, Michael/Jakobsen, Arnt L./Hvelplund Jensen, Kristian T. (2008): Modelling human translator behaviour with user-activity data. In: Proceedings of the 12th EAMT Conference. Hamburg, S. 21-26.

Čulo, Oliver et al. (2008): Empirical studies on language contrast using the English-German comparable and parallel CroCo corpus. In: Calzolari, Nicoletta et al. (Hg.): Workshop abstracts: Sixth International Conference on Language Resources and Evaluation (LREC), May 26-June 1, 2008, Marrakech. Paris, S. 47-51.

Hansen-Schirra, Silvia (2008): Interactive reference grammars: Exploiting parallel and comparable treebanks for translation. In: Yuste Rodrigo, Elia (Hg.): Topics in language resources for translation and localisation. (= Benjamins Translation Library - EST Subseries 79). Amsterdam, S. 23-37. 
Hansen-Schirra, Silvia/Neumann, Stella/Steiner, Erich (2012): Cross-linguistic corpora for the study of translations. Insights from the language pair English-German. (= Text, Translation, Computational Processing 11). Berlin/New York.

Hawkins, John A. (1986): A comparative typology of English and German. Unifying the contrasts. London.

Jakobsen, Arnt L./Hvelplund Jensen, Kristian T. (2008): Eye movement behaviour across four different types of reading task. In: Göpferich, Susanne/Jakobsen, Arnt L./Mees, Inger M. (Hg.): Looking at eyes: Eye-tracking studies of reading and translation processing. (= Copenhagen Studies in Language 36). Kopenhagen, S. 103-124.

Just, Marcel A./Carpenter, Patricia A. (1980): A theory of reading: From eye fixations to comprehension. In: Psychological Review 87, S. 329-354.

König, Ekkehard/Gast, Volker (2007): Understanding English-German contrasts. (= Grundlagen der Anglistik und Amerikanistik 29). Berlin.

Krein-Kühle, Monika (2001): Towards a methodology for the investigation of equivalence in scientific and technical translation. In: Proceedings of the 1st International Conference on Specialized Translation, 2-4 March 2000, Barcelona. Barcelona, S. 79-82.

Newmark, Peter (1988): A textbook of translation. New York.

Olohan, Maeve/Baker, Mona (2000): Reporting that in translated English: Evidence for subconscious processes of explicitation? In: Across Languages and Cultures 1, S. 141-172.

Rabe, Jennifer (2012): Lesen Sie die Packungsbeilage? - Eine Eye-Tracking-Rezeptionsstudie von Verbrauchern und (semi-)professionellen Fachübersetzern. MA Thesis. Germersheim.

Rayner, Keith (1998): Eye movements in reading and information processing: 20 years of research. In: Psychological Bulletin 124, S. 372-422.

Rohdenburg, Günter (1974): Sekundäre Subjektivierungen im Englischen und Deutschen: Vergleichende Untersuchungen zur Verb- und Adjektivsyntax. (= PAKS-Arbeitsbericht 8). Bielefeld.

Toury, Gideon (1995): Descriptive translation studies and beyond. (= Benjamins Translation Library 4). Amsterdam.

Vinay, Jean-Paul/Darbelnet, Jean (1995): Comparative stylistics of French and English: A methodology for translation. (= Benjamins Translation Library 11). Amsterdam. 